\title{
Low-energy Bluetooth for detecting real-world penetrance of bystander naloxone kits: a pilot study
}

\author{
Jeffrey T. Lai \\ University of Massachusetts \\ Medical School \\ jeffrey.1ai@umassmemorial.org \\ Edward W. Boyer \\ Harvard Medical School \\ eboyer@bwh.harvard.edu
}

\author{
Brittany P. Chapman \\ University of Massachusetts \\ Medical School \\ brittany.chapman@umassmed.edu
}

\author{
Katherine L. Boyle \\ Harvard Medical School \\ klboyle@bidmc.harvard.edu
}

Peter R. Chai

Harvard Medical School

pchai@bwh.harvard.edu

\begin{abstract}
Opioid overdose is a growing public health emergency in the United States. The antidote naloxone must be administered rapidly after opioid overdose to prevent death. Bystander or "take-home", naloxone programs distribute naloxone to opioid users and other community members to increase naloxone availability at the time of overdose. However, data describing the natural history of takehome naloxone in the hands of at-risk individuals is lacking. To understand patterns of naloxone uptake in at-risk users, we developed a smart naloxone kit that uses low-energy Bluetooth (BLE) to unobtrusively detect the transit of naloxone through a hospital campus. In this paper, we describe development of the smart naloxone kit and results from the first 10 participants in our pilot study.
\end{abstract}

\section{Introduction}

Heroin overdose deaths have more than doubled from 2000 to 2014, triggering the growth of harmreduction programs aimed at 1) training non-medical personnel to recognize and respond to opioid overdoses, and 2) dispensing overdose rescue kits containing naloxone, the antidote for opioid overdose, to at-risk individuals and their friends and family from community and emergency department sites.[3,6,9,12,15] Despite the growth of "bystander" naloxone distribution programs and observations of a decrease in opioid overdose deaths in communities where they have been implemented, overdose deaths continue to increase.[16] The Centers for Disease Control (CDC) reported 33,091 individuals died from opioid overdose in 2015, a $15.6 \%$ increase since
2014.[13] Unfortunately, rigorous data delineating what happens to these overdose rescue kits once distributed to heroin users are lacking. Understanding the patterns of naloxone distribution and use in a community through advanced technology-based monitoring can guide strategies to improve naloxone distribution programs.[8]

The introduction of a technology-based monitoring system that can easily integrate into a standard overdose rescue kit provides the ability to directly ascertain the real-time location of naloxone rescue kits. By providing insights into the patterns of naloxone distribution and use in a community, these technology-enabled naloxone kits can provide valuable information on real-world uptake of bystander naloxone by active heroin users and help to optimize naloxone distribution programs. Furthermore, this information can guide the development of real-time mobile technology interventions for these at-risk populations.

Discovering the fate of naloxone rescue kits can be accomplished through unobtrusive low-energy Bluetooth (BLE) technology. Similar to monitoring medical equipment or patients as they transit through a hospital system, BLE technology incorporated into naloxone rescue kits dispensed from an emergency department (ED) setting can provide insight into the uptake of naloxone kits by heroin users and the availability of these kits for use in the community.[10,14]

In this pilot study, we deployed a naloxone rescue kit with an integrated BLE beacon ("smart naloxone kit") to help determine the community penetrance of ED distributed naloxone.

\section{The smart naloxone kit}


The "smart naloxone kit" consists of a standard Food and Drug Administration (FDA)-approved intranasal naloxone syringe combined with a BLE identification tag. As smart naloxone kits exit the emergency department, they enter detection zones of strategically placed BLE readers which collect and $\log$ the transit of smart naloxone kits through specific locations on a hospital campus (Figures $1 \& 2$ ). Data from BLE readers indicate the proportion of smart naloxone kits that become available for use in the community.
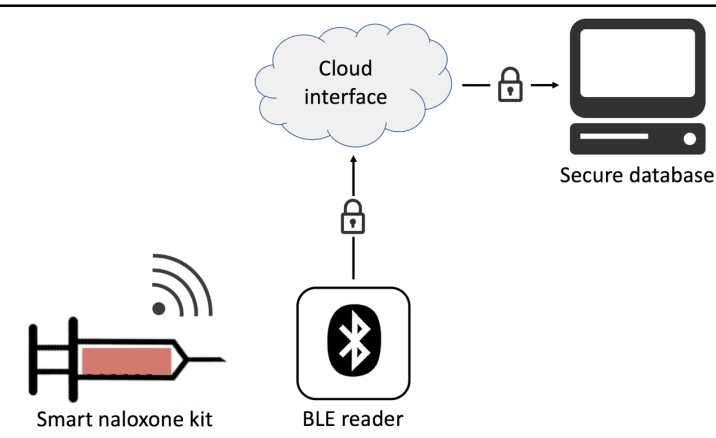

Figure 1. The BLE-enabled smart naloxone kit enters the detection zone of a BLE reader, transmitting a unique code to a cloud-based interface. Data is subsequently downloaded to a secure database, indicating the transit of the smart naloxone kit.

\subsection{Low-energy Bluetooth identification beacons}

BLE beacons $(36 \mathrm{~mm} \times 23 \mathrm{~mm} \times 4 \mathrm{~mm})$ powered by an integrated battery actively transmit a preprogrammed unique identifier code. BLE beacons have a battery life of three months. Beacons transmit pre-programmed identifiers using 2.4 gigahertz $(\mathrm{GHz})$ radiofrequency waves, the same frequency employed by most $\mathrm{WiFi}$ routers and cordless telephones.[5] By using a set $2.4 \mathrm{GHz}$ frequency, BLE beacons avoid interference from conventional medical equipment like intravenous pumps, cardiac pacemakers and insulin pumps.

\subsection{Low-energy Bluetooth systems do not affect biological systems}

Theoretical concerns have been raised regarding the ability of radiofrequency waves to alter the molecular composition and efficacy of xenobiotics.[2] We believe this concern is unfounded. Studies exposing radiofrequency identification (RFID) systems, a similar technology to BLE but employing slightly different frequencies, have shown no alteration in the stereochemistry or molecular composition of xenobiotics.[1,2] Additionally, RFID is increasingly utilized in pharmaceutical manufacturing, underscoring the safety of using this technology near xenobiotics.[7]

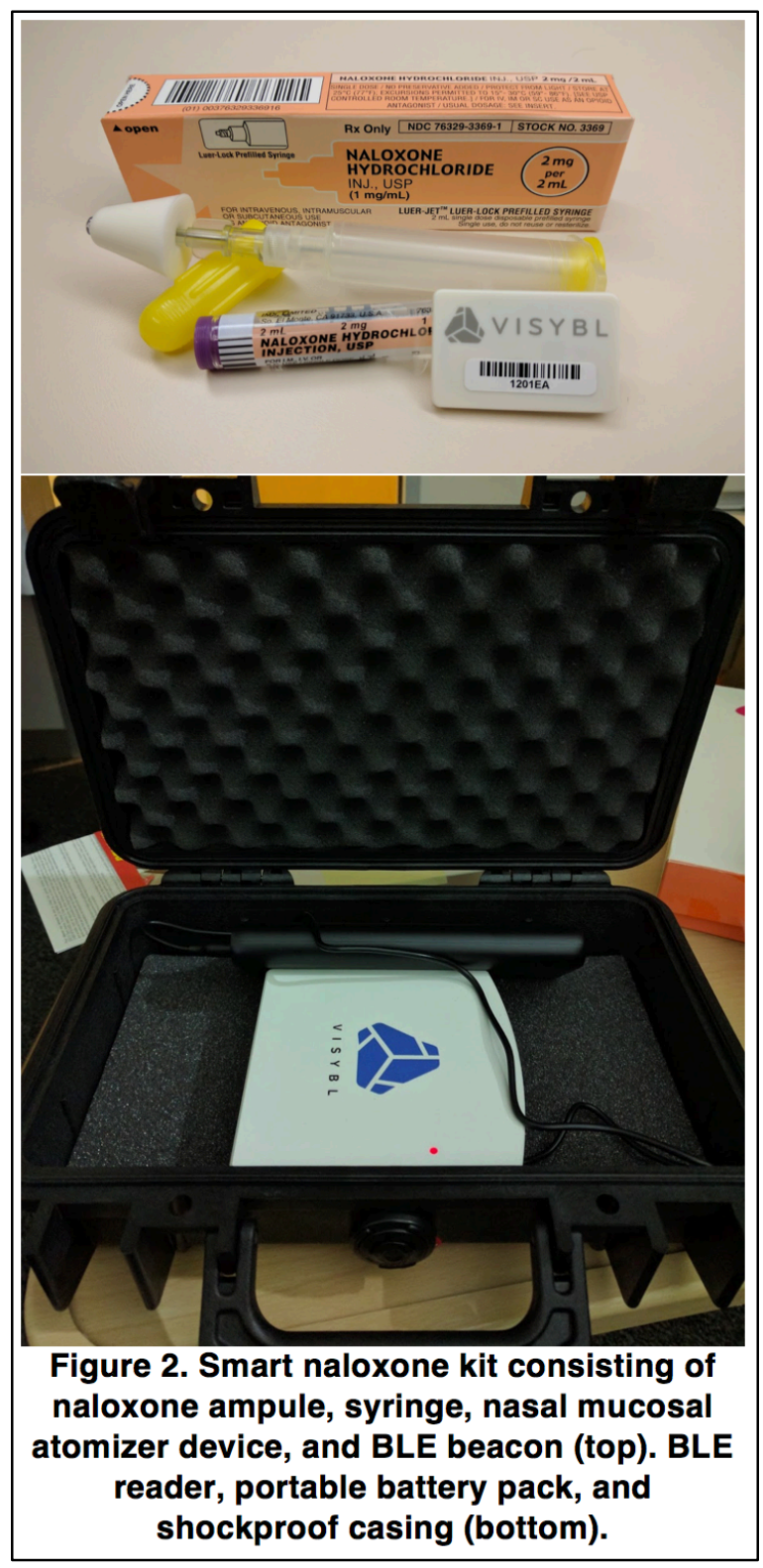

\subsection{Low-energy Bluetooth readers}

BLE readers, approximately the size of a wireless router, serve as a relay station for information transmitted by BLE beacons: when a BLE beacon enters the detection radius of a BLE reader, the beacon transmits pre-recorded data 
including the specific beacon identification number. This information is captured by the BLE reader and securely stored on its on-board, encrypted memory. The BLE reader also transmits BLE beacon transit data to a secure cloud-based interface. BLE readers have a detection radius of 20 meters and can penetrate clothing and metal, therefore allowing detection of smart naloxone kits carried in pockets, cars, or buses. The readers are powered by rechargeable batteries that last for 24 hours, allowing them to be affixed to telephone poles or light posts without a grounded power source. The readers are housed in locked weatherproof, shockproof casings built to military specification (Figure 2).

\subsection{BLE reader placement}

BLE readers were positioned at strategic locations around the hospital campus, corresponding to the avenues of pedestrian and vehicular egress that patients utilize when they are discharged from the ED: the ED registration area, the ambulance bay exit, the pedestrian walkway to the bus stop, and the two main roads for automobile traffic. These locations were chosen to capture participants as they exited the hospital campus 1) by walking or driving along the main roads, 2) by ambulance, 3) by public transit (Figure 3).

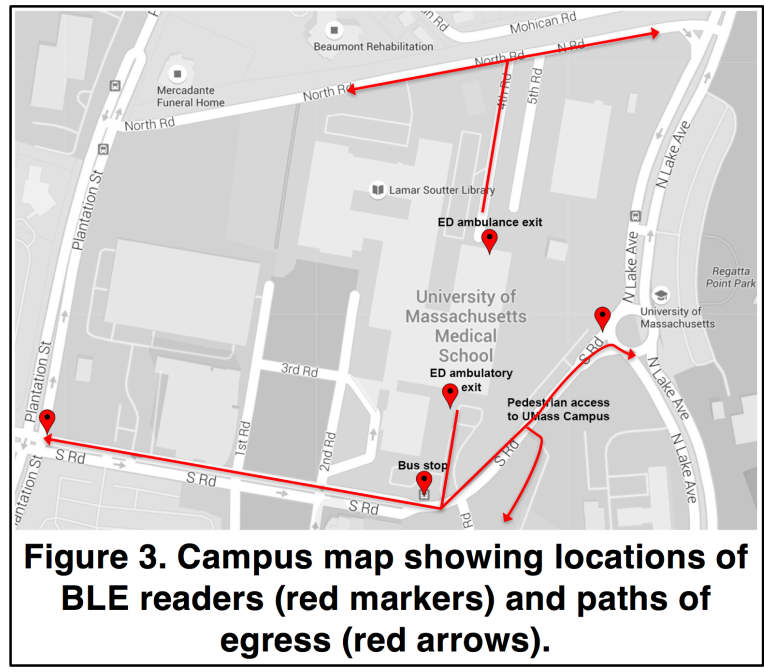

By selecting BLE readers with adequate range to cover major road intersections and power to penetrate cars, we can detect the transit of smart naloxone kits off of a hospital campus. This approach is highly scalable-rapid deployment of BLE readers throughout a community in response to an escalating heroin epidemic can help public health officials and researchers discover hotspots of naloxone as a marker for heroin use, and target interventions to these key geographic areas.

\section{Methods}

\subsection{Design considerations}

The smart naloxone kit was designed to be discreet to maximize acceptance by heroin users. Placing unobtrusive BLE tags within the carton containing the syringe, ampule and nasal atomizer ensures the ability to locate smart naloxone kits without obvious indicators that kits are being tracked. Affixing the monitoring device to a critical component such as the naloxone syringe was considered to address the scenario where a participant might keep the medication and atomizer while discarding the box prior to leaving the hospital, but this was not done out of concern that it might interfere with the efficient delivery of naloxone in an emergency situation.

Similarly, BLE readers were selected because they provided the necessary combination of detection range and penetrance around metal and human bodies (which can interfere with the performance of other modalities such as RFID), allowing for the detection of tagged kits carried in various ways, including in pockets, bags, cars, or buses.

Additionally, using advanced technology to detect devices carried by patients with active drug use may pose ethical concerns.[11] The smart naloxone kit does not track patients continuously. Instead, it utilizes a BLE tag that transmits a unique 6 character identification code that is detected by a compatible BLE reader. Thus, the BLE system does not provide continuous location data; rather it captures discrete episodes of movement through BLE reader detection zones.

Although the system does not continuously track patients, security remains an important consideration. Radiofrequency ID systems such as BLE can be penetrated through passive eavesdropping, signal jamming, or reader tampering.[11] Several methods are employed to ensure the security of the BLE system. First, BLE utilizes advanced encryption technologies, the same standard used by the US government.[5] Second, rather than using patient identifiers such as name, birthdate, or medical record number, the BLE tag utilizes the previously mentioned 6 character code to identify itself to a BLE reader, minimizing any threat to privacy or confidentiality should the BLE system be compromised. Third, once detected by the reader, the BLE tag identifier is transmitted via secure $\mathrm{WiFi}$ or cellular hotspot connection to a secure password 
protected interface; alternatively, if no internet connection is available, the BLE tag information is stored locally on the reader using encrypted internal storage until such a time that an internet connection is available. Finally, careful selection of BLE reader placement prevents interference from other devices utilizing similar radiofrequency transmissions, and physical security measures such as locks and cables minimize tampering with the devices.

\subsection{Technology acquisition and verification}

BLE beacons (Visybl AssetBeacons) and readers (Visybl CloudNodes) were purchased from the manufacturer (Visybl Inc., Germantown, MD, USA). Field testing was performed using BLE readers positioned around the hospital campus, and BLE beacons in various configurations to simulate real-life situations in which a smart naloxone kit might be carried (e.g. in medication cartons, in shirt and pants pockets, inside a moving vehicle). The author (JL) carried a BLE beacon through detection zones for all readers to ensure reliable signal capture.

\subsection{Study design}

This was a prospective pilot study utilizing a convenience sample of participants presenting to the ED with a heroin-related complaint. Participants were eligible for the study if they were 18 years of age or older, English-speaking, able to provide written informed consent, and in possession of a mobile phone with text messaging capability. Potential subjects were excluded if they were pregnant, prisoners or in police custody, or presenting with a primary psychiatric complaint.

Participants were enrolled, provided written consent, and underwent a standardized training session on using naloxone and the functionality of the smart naloxone kit. Consenting participants were given a brief training on recognizing signs of heroin and opioid overdose in addition to the assembly and operation of the smart naloxone kit, in accordance with validated methods of naloxone teaching (prescribetoprevent.com). Next, participants were given an overview of the BLE technology utilized in the kit. The individual BLE beacon was activated, verified to be operational, and sealed in the naloxone kit with double sided tape. Contact information was obtained from participants, including personal phone number and phone number of a friend or family member, to maximize our ability to contact subjects for follow up.

As participants departed from the ED, transit of the smart naloxone kit off the hospital campus was detected by the BLE readers and accessed by batch download using a secure cloud-based interface and recorded by study staff.

Subjects were contacted via text message to confirm accuracy of the technology on days 7, 10, and 13. If there was no response, subjects were contacted via phone call on days 7 and 10. If there was no response to these phone calls, then subjects' alternate contacts were called. If no response from subjects or alternate contacts at 14 days, the subject was considered lost to follow up.

The primary outcome for this study was demonstrating the feasibility of utilizing a BLE system to observe the transit of naloxone off a hospital campus. Secondary outcomes included observing the number of kits leaving the hospital campus and whether these kits were utilized by participants.

\section{Results}

During the study period, a total of 102 individuals presented to the ED with a heroin overdose or heroin-related complaint. Of these, 10 individuals were consented, and no individuals declined participation. Ten individuals completed the study over the 3-month study period. Median age was 29 years, ten identified as Caucasian, nine participants identified as male, and one participant identified as female. Seven presented to the ED after a heroin overdose while three presented after a heroin-related complaint (Table 1).

Table 1. Participant demographics. OD denotes overdose.

\begin{tabular}{|c|c|c|c|c|}
\hline ID & Age & Sex & Race & $\begin{array}{c}\text { Triage } \\
\text { Complaint }\end{array}$ \\
\hline $\mathbf{r}$ & 39 & M & White & Withdrawal sx \\
\hline$\square$ & 24 & M & White & OD \\
\hline$\square$ & 20 & M & White & OD \\
\hline$\square$ & 28 & M & White & OD \\
\hline$\Sigma$ & 31 & M & White & Detox \\
\hline $\boldsymbol{\square}$ & 29 & M & White & OD \\
\hline$\square$ & 29 & M & White & Eval \\
\hline$\Delta$ & 29 & M & White & Heroin OD \\
\hline$\square$ & 28 & M & White & OD \\
\hline$\triangleright$ & 29 & F & White & OD \\
\hline
\end{tabular}




\subsection{Smart naloxone kit uptake and distribution}

All 10 individuals accepted the smart naloxone kit and were trained to recognize signs of an opioid overdose and administer naloxone. $70 \%$ of smart naloxone kits $(\mathrm{N}=7)$ were detected by BLE readers positioned at egress points from the hospital campus. Two of the participants whose smart naloxone kits were detected by the ED ambulatory exit reader but not by the readers positioned along the egress paths reported during follow-up that they carried the smart naloxone kit off campus (Figure 4). This was likely due to technical errors in which the readers were not powered on. Of the four participants we successfully contacted, one reported having utilized the smart naloxone kit to reverse an opioid overdose. Unfortunately, $60 \%$ of participants $(\mathrm{N}=6)$ were lost to follow up.

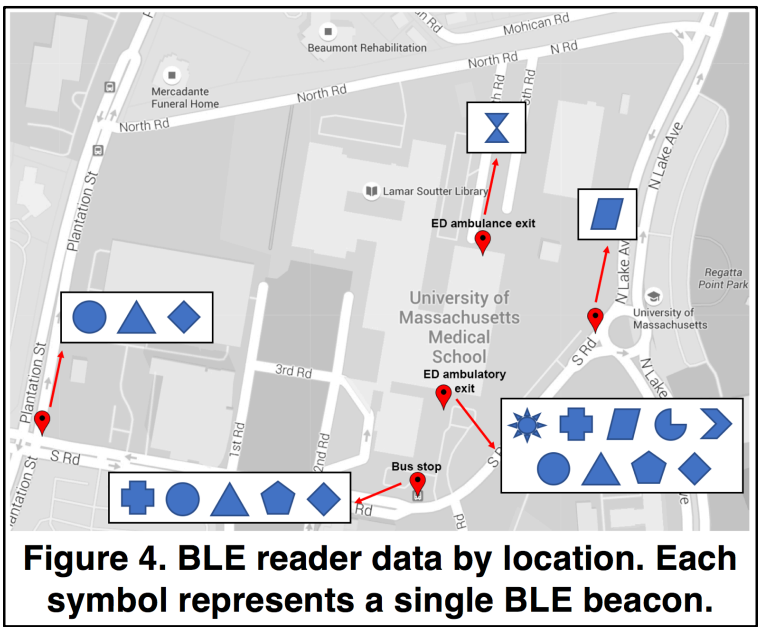

\section{Discussion}

Our data shows that a majority of individuals accept and carry ED distributed naloxone off a hospital campus and into the community. A majority of participants carried the smart naloxone kits with them on discharge from the ED, suggesting that heroin users recognized the dangers of overdose, were willing to carry naloxone, and were willing to use smart naloxone kits to inform us about their patterns of naloxone uptake. This pilot study demonstrates that the smart naloxone kit is an acceptable and feasible platform to improve understanding of naloxone uptake and ultimately acceptance among individuals with active substance use.

Our smart naloxone kits were able to provide passively generated evidence of naloxone uptake and retention through a hospital campus. This pilot provides a template for mini smart naloxone kit systems to be deployed in conjunction with new or existing naloxone distribution programs to assess the actual uptake of naloxone. Expansion of these concepts could lead to strategic placement of BLE readers around known overdose locations in a city to determine if distributed naloxone first moves out of the naloxone distribution site and if the distributed kits are found in areas with overt overdose.

Engaging heroin users to understand factors leading to acceptance or non-acceptance of advanced monitoring devices is essential in the development of future iterations of smart naloxone kits.[4] Successful deployment of a smart naloxone distribution program is likely contingent upon actively involving heroin users in their design and providing reassurances that privacy (e.g. location data not shared with law enforcement) is held paramount.

\subsection{Limitations}

Although our concept is highly scalable, it has several limitations. Our pilot study was aimed at assessing feasibility of using BLE to detect naloxone as it exits the site of distribution from our ED at a single hospital campus. However, this study phase is crucial to determining if patients will accept this technology and distribute it throughout an urban area. Further studies will require municipal participation to place BLE readers at additional locations, expanding the potential detection of smart naloxone kits in our community.

Regrettably, we were unable to contact six participants: phone numbers from five participants either were disconnected or were unanswered, and one participant was unable to be reached due to constraints of his treatment program.

\subsection{Future directions}

BLE labeled naloxone provides a system that is easily and quickly deployable that can be leveraged to respond to the rising opioid epidemic across the United States. An advantage of utilizing BLE-based tracking platform is that Bluetooth technology is ubiquitous: nearly all smartphones are equipped with Bluetooth radios and can functionally serve as BLE readers. Thus, any Bluetooth-enabled mobile phone can be set up to detect our smart naloxone kit. This "crowdsourcing" of BLE tag detection offers a costeffective method to vastly expand the ability to detect a smart naloxone kit in an urban environment where smartphones are omnipresent. 


\section{Conclusion}

A smart naloxone kit comprised of BLE-labeled nasal naloxone syringe and strategically placed BLE readers can provide insight into natural uptake patterns of naloxone among individuals at risk for heroin overdose. This information will allow for improvement in planning community-based interventions to prevent deaths from opioid overdose.

\section{Funding source}

This project was funded by the Medical Toxicology Foundation/American College of Medical Toxicology Innovative Research and Teaching Award.

\section{References}

[1] Acierno, R., Carata, E., De Pascali, S.A., et al. Potential effects of RFID systems on biotechnology insulin preparation: A study using HPLC and NMR spectroscopy. IEEE (2010), 198-203.

[2] Acierno, R., De Pascali, S.A., Fanizzi, F.P., et al. Investigating potential effects of RFID systems on the molecular structure of the human insulin. IEEE (2010), 192-196.

[3] Beletsky, L., Rich, J.D., and Walley, A.Y. Prevention of Fatal Opioid Overdose. JAMA 308, 18 (2012), 1863-1864.

[4] Birnbaum, F., Lewis, D., Rosen, R.K., and Ranney, M.L. Patient Engagement and the Design of Digital Health. Academic Emergency Medicine 22, 6 (2015), 754-756.

[5] Bluetooth SIG. Bluetooth specification version 4.2. (2014), 1-2772.

[6] Boyer, E.W. Management of opioid analgesic overdose. The New England journal of medicine 367, 2 (2012), 146-155.

[7] Catarinucci, L., Colella, R., De Blasi, M., Patrono, L., and Tarricone, L. Enhanced UHF RFID tags for drug tracing. Journal of medical systems 36, 6 (2012), 3451-3462.

[8] Compton, W.M., Volkow, N.D., Throckmorton, D.C., and Lurie, P. Expanded access to opioid overdose intervention: research, practice, and policy needs. Annals of internal medicine 158, 1 (2013), 65-
66.

[9] Doe-Simkins, M., Walley, A.Y., Epstein, A., and Moyer, P. Saved by the nose: bystander-administered intranasal naloxone hydrochloride for opioid overdose. American journal of public health 99, 5 (2009), 788-791.

[10] Kim, D.-S., Kim, J., Kim, S.-H., and Yoo, S.K. Design of RFID based the Patient Management and Tracking System in hospital. Conference proceedings $\therefore$... Annual International Conference of the IEEE Engineering in Medicine and Biology Society. IEEE Engineering in Medicine and Biology Society. Annual Conference 2008, (2008), 1459-1461.

[11] Rosenbaum, B.P. Radio frequency identification (RFID) in health care: privacy and security concerns limiting adoption. Journal of medical systems 38, 3 (2014), 19.

[12] Rudd, R.A., Aleshire, N., Zibbell, J.E., and Gladden, R.M. Increases in Drug and Opioid Overdose Deaths — United States, 2000-2014. MMWR. Morbidity and mortality weekly report 64 , 50-51 (2016), 1378-1382.

[13] Rudd, R.A., Seth, P., David, F., and Scholl, L. Increases in Drug and Opioid-Involved Overdose Deaths - United States, 2010-2015. MMWR. Morbidity and mortality weekly report 65, 5051 (2016), 1445-1452.

[14] Shirehjini, A.A.N., Yassine, A., and Shirmohammadi, S. Equipment location in hospitals using RFID-based positioning system. IEEE transactions on information technology in biomedicine : a publication of the IEEE Engineering in Medicine and Biology Society 16, 6 (2012), 10581069.

[15] Walley, A.Y., Doe-Simkins, M., Quinn, E., Pierce, C., Xuan, Z., and Ozonoff, A. Opioid overdose prevention with intranasal naloxone among people who take methadone. Journal of substance abuse treatment 44, 2 (2013), 241-247.

[16] Walley, A.Y., Xuan, Z., Hackman, H.H., et al. Opioid overdose rates and implementation of overdose education and nasal naloxone distribution in Massachusetts: interrupted time series analysis. BMJ (Clinical research ed.) 346, jan30 5 (2013), f174f174. 